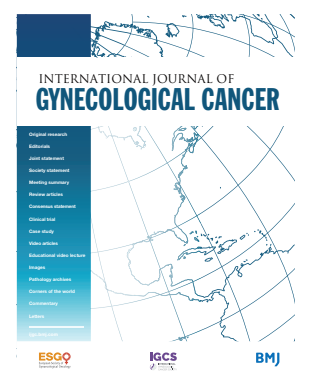

Correspondence to Dr Sol Gómez Rodrigo, Ginecología, Hospital Universitario de Maternidad y Neonatología, Cordoba, Argentina; solgomez90@ hotmail.com

Accepted 21 December 2020

Check for updates

(C) IGCS and ESGO 2021. No commercial re-use. See rights and permissions. Published by BMJ.

To cite: Gómez Rodrigo S, Calderon J, Dionisi JN, et al. Int J Gynecol Cancer 2021;31:784-788.

\title{
Cervical cancer in pregnancy at various gestational ages
}

\author{
Presenters: \\ Sol Gómez Rodrigo \\ Ginecología, Hospital Universitario de Maternidad y Neonatología, Cordoba, Argentina \\ Josefina Calderon \\ Ginecología, Hospital Universitario de Maternidad y Neonatología, Cordoba, Argentina \\ Jorge Nicolas Dionisi \\ Ginecología, Hospital Universitario de Maternidad y Neonatología, Cordoba, Argentina \\ Agostina Santi \\ Ginecología, Hospital Universitario de Maternidad y Neonatología, Cordoba, Argentina \\ Jose Maria Mariconde \\ Ginecología, Hospital Universitario de Maternidad y Neonatología, Cordoba, Argentina \\ Otilio Daniel Rosato \\ Ginecología, Hospital Universitario de Maternidad y Neonatología, Cordoba, Argentina
}

Frédéric Amant

Division of Gynecologic Oncology, Department of Obstetrics and Gynecology, University Hospitals Leuven, Katholieke Universiteit Leuven, Leuven, Belgium

\section{CASE 1}

A 28-year-old patient $G_{1} P_{0}$ was referred to the University Hospital of Maternity and Neonatology at 33 weeks of pregnancy with unremarkable medical, surgical, or family history. She presented with a diagnosis of moderately differentiated invasive adenocarcinoma of the cervix. During prenatal care, in a small rural hospital at the time of 28 weeks' gestation she had presented with abnormal vaginal bleeding. An exophytic cervical tumor measuring $3 \mathrm{~cm}$ diameter with no evidence of parametrial or vaginal involvement was found on physical examination. A biopsy was performed, and this was consistent with a moderately differentiated invasive adenocarcinoma of the cervix (FIG0 2018 stage: IB2). Pelvic MRI without contrast was performed at 31 weeks. At the time of her presentation to our hospital at 33 weeks' gestation, in the University Hospital of Maternity and Neonatology, the physical examination was consistent with the previously described tumor in the cervix measuring $3 \mathrm{~cm}$ in diameter, taking into account the normal physiological changes in a gravid uterus.

The pelvic MRI showed an intrauterine gestation with a tumor measuring $19 \times 16 \times 10 \mathrm{~mm}$ on the anterior lip of the uterine cervix. There was no evidence of lymph node involvement. In addition, there was no parametrial or vaginal compromise. The urinary bladder and urethra appeared normal. No iliac or inguinal adenopathy was documented (Figure 1).
Dr Amant: What would be your recommendation at this time based on the gestational age of the patient and the stage of the cervical cancer?

Cervical cancer diagnosed during pregnancy is the most challenging situation since the pregnant uterus itself is involved. Where possible, standard treatment is applied during pregnancy. ${ }^{1}$ Individualization according to gestational age and cervical cancer stage impacts on the optimal approach. ${ }^{12}$ Based on data collected by the International Network on Cancer Infertility and Pregnancy (INCIP), ${ }^{3}$ the prognosis is not worse when compared with a non-pregnant population. ${ }^{4}$ At 33 weeks' gestational age, surgical options are technically impossible (pelvic lymphadenectomy) or associated with too high blood loss and risk of fetal loss (trachelectomy) and hence contraindicated. Two treatment options therefore remain. Both a conservative approach or initiation of chemotherapy to help reach fetal maturity, which is defined as a delivery after 37 weeks' gestational age. There is a consensus that a delay of 6 weeks is unlikely to affect the maternal prognosis and is found acceptable in this setting for the benefit of the child. Alternatively, chemotherapy at 33 weeks would consist of one 3-weekly regimen or 3-4 weekly regimens of paclitaxel-carboplatin. Elective Cesarean section is performed after hematologic recovery which is respectively in the order of 1 to 3 week(s). A second 3 -weekly dose at 36 weeks' gestational age is therefore not recommended. 


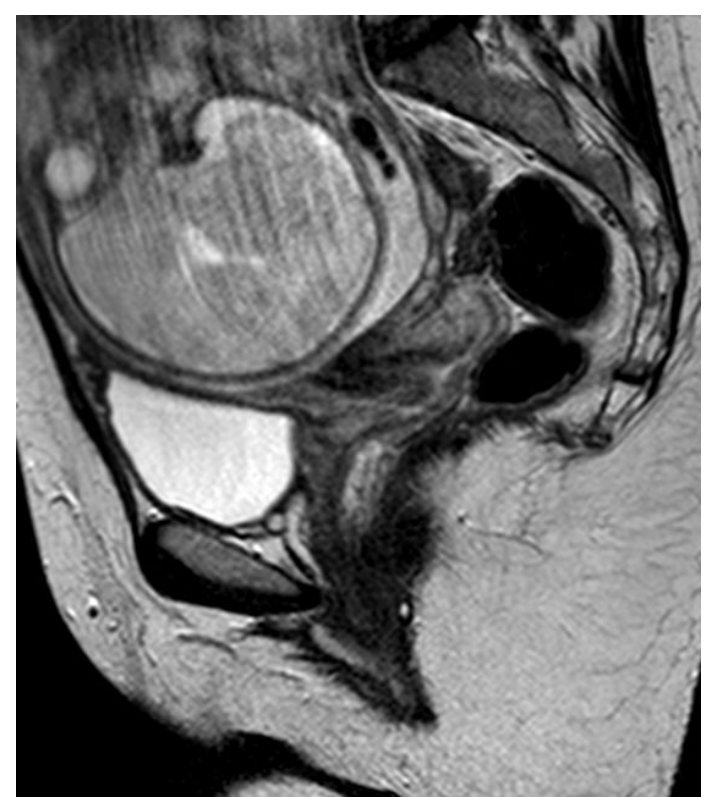

Figure 1 Pelvic MRI without contrast at 31 weeks' gestation.

The case was discussed by the Tumor Committee (Obstetrics, Neonatology, Gynecology, Pathology, and Oncology) that recommended expectant management awaiting fetal maturity at 36 weeks (with one single dose of betamethasone $12 \mathrm{mg}$ ) and a Cesarean-radical hysterectomy at the time of delivery. The pregnancy continued with no other complications. At 36 weeks' gestation a Cesarean section was performed through a Cherney incision. A male child was delivered (Apgar 8/9, $2830 \mathrm{gm}$ ). After delivery, a type $\mathrm{C} 1$ radical hysterectomy (Morrow-Querleu) with pelvic lymphadenectomy and bilateral oophoropexy was performed: the latter is routinely performed in our institution. Lymphadenectomy according with anatomical landmarks was performed (superior limit was at the common iliac vessels; inferior limit in the circumflex iliac vein; and medial and deep limit was the obturator nerve).

\section{Dr Trezza}

There was a cervical tumor measuring $3 \times 1 \mathrm{~cm}$ with infiltration of the inner half of the cervix ( $50 \%$ infiltration) and $10 \mathrm{~mm}$ of invasion. Vaginal cuff $(25 \mathrm{~mm})$, left parametrial $(20 \times 13 \mathrm{~mm})$, and right parametrial $(20 \times 15 \mathrm{~mm})$ margins were free of disease. A total of five pelvic lymph nodes were obtained and all were negative for disease. Moderately differentiated invasive adenocarcinoma was diagnosed (Figure 2A\&B).

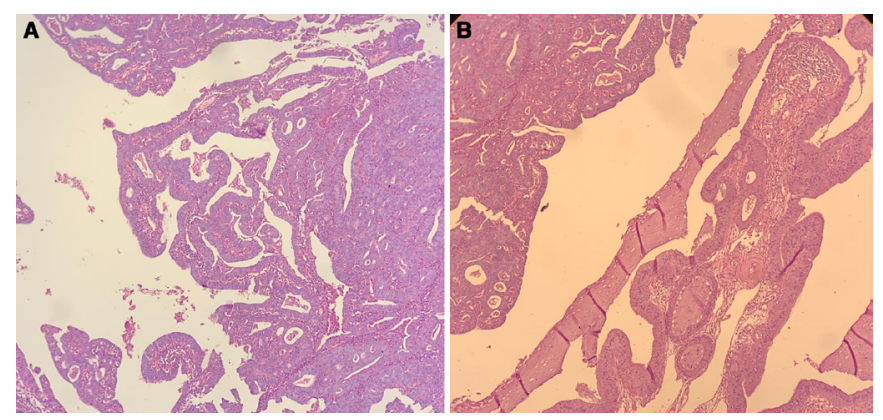

Figure 2 (A,B) H\&E showing invasive adenocarcinoma grade 2 .
After surgery, the case was discussed by the Tumor Committee, and although the patient did not meet standard criteria for postoperative radiotherapy, based on the fact that only five lymph nodes were obtained, the group recommended intensity modulated radiotherapy followed by brachytherapy. The patient is free of disease 11 months after her surgery.

\section{Dr Amant: First, please comment on the role of Cesarean- radical hysterectomy vs delayed surgery. Second, based on the findings on the postoperative pathology, what would have been your recommendation?}

Reoperation in the same surgical field is best executed from 6 weeks onwards after the first surgery. By that time, the postoperative increased vascularization that allows wound healing is normalized. Thus, a radical hysterectomy can be performed from 6 weeks onwards after Cesarean section. But this waiting time can be avoided since a Cesarean-radical hysterectomy is technically possible and is, in experienced hands, not associated with increased blood loss. At first an obstetric team performs the Cesarean section, extracts the placenta, and closes the uterus. Then, an oncological team proceeds with the radical hysterectomy. Although blood vessels are more congested immediately after delivery, surgical plains are sometimes even easier to dissect and contribute to safe surgery with experienced surgeons.

The pathology report only describes five harvested pelvic lymph nodes. I would ask for a revision of the specimen and search for more nodes, since the most important anatomical landmarks for a bilateral pelvic lymphadenectomy were dissected, which should lead to a significant higher number of retrieved nodes. Five lymph nodes is far below what is believed to be a complete pelvic lymphadenectomy and it is unlikely that this number will be corrected significantly after pathological re-evaluation. Most specialists believe this number of nodes should vary with approximately 20 lymph nodes for both sides together. Therefore, indeed, postoperative IMRT is indicated to correct for an incomplete pelvic lymphadenectomy and to avoid pelvic sidewall recurrence.

\section{CASE 2}

A 41-year-old patient $\mathrm{G}_{6} \mathrm{P}_{4}$ presented to the University Hospital of Maternity and Neonatology with an abnormal routine Pap screening and a subsequent diagnosis of a squamous cell moderately differentiated cervical invasive carcinoma confirmed by biopsy. The patient had a past surgical history significant for appendectomy, cholecystectomy, and two uterine curettages. A $2 \mathrm{~cm}$ tumor on the anterior lip of the cervix with no evidence of parametrial or vaginal involvement was found on physical examination (FIGO 2018 stage IBI). An abdominal and pelvic MRI was ordered. The study revealed heterogeneity in the anterior aspect of the uterine cervix (dimensions not provided by radiologist). In the anterior wall, there was significant thickening of the myoendometrial junction area. Diffuse pseudonodular appearance corresponded to marked ademomyosis. No suspicious lymph nodes or parametrial involvement and no evidence of tumor infiltration in the vagina was noted.

Given the results of the MRI and the last menstrual period, an ultrasound was requested which confirmed that the patient was 6 weeks pregnant (also confirmed by serum $\beta H C G$ ). The case was discussed by the Tumor Committee and the following options 


\section{Case study}

were entertained. If the patient wished to preserve her pregnancy, our recommendation would be to proceed with a laparoscopic lymphadenectomy, if this was negative for disease, then a radical trachelectomy at 12 weeks of gestation was to be recommended. If positive nodes were found at the time of lymphadenectomy, another option was neoadjuvant chemotherapy after 12 weeks and Cesarean section at 32 weeks followed by radical hysterectomy. Lastly, the option of expectant management up to approximately 32 weeks and Cesarean section at the time of confirmation of fetal maturity was also considered. We also discussed that if the patient decided to end her pregnancy then proceeding with recommendation of pregnancy termination (Argentina legal abortion according to Article 86 of legislation) plus type $\mathrm{C} 1$ radical hysterectomy with ovarian preservation.

\section{Dr Amant: Please provide your feedback with regards to these recommendations}

Cervical cancer early in pregnancy is one of the major challenges in this field. Apart from the vulnerability of early human life, the time needed to reach fetal maturity is significant. Although we gained more experience in the last two decades, cervical cancer treatment during pregnancy still is an experimental setting. This experimental nature adds to the importance of patients' motivation to proceed with the pregnancy. In this case, a mother of four children suffering from a cervical cancer stage lb1 diagnosed at 6 weeks' pregnancy, needs to decide on a still experimental approach or final treatment that includes loss of her pregnancy.

In case the mother would be very motivated to preserve her pregnancy, several options need to be discussed. I would not opt for a conservative approach, even in case lymph nodes are negative for disease, since the time frame needed to obtain fetal maturity is too long. Although one study showed that in lymph node negative patients, a waiting period of 17 weeks was safe, ${ }^{2}$ the time span until fetal maturity in this case is too large to be certain about its safety. Given the location of a $2 \mathrm{~cm}$ tumor on the anterior cervix, one option could be to start with an (endoscopic) pelvic lymphadenectomy. Positive nodes would indicate high-risk disease and need for final treatment and fetal loss. ${ }^{1}$ As an alternative to radical trachelectomy that is associated with significant blood loss and risk of fetal loss, a simple trachelectomy (cervical amputation) could be discussed. ${ }^{5}$ Non-pregnant patients with this tumor would have qualified for the SHAPE trial where patients were randomized between radical vs standard hysterectomy. Although final data are not mature yet, retrospective studies indicate that node negative cervical cancers $<2 \mathrm{~cm}$ have $\mathrm{a}<1 \%$ chance for parametrial involvement. ${ }^{6} \mathrm{MRI}$ is very important here to assess technical feasibility

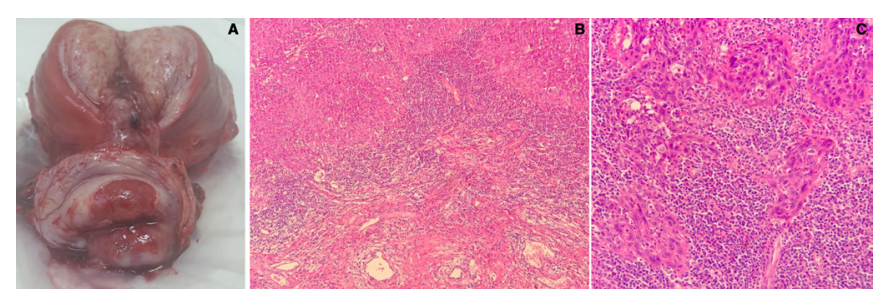

Figure 3 (A) Hysterectomy specimen, (B,C) microscopy H\&E: squamous cell moderately differentiated cervical invasive carcinoma. and chance for simple trachelectomy/ cervical amputation with free margins. In case this is not considered safe, neoadjuvant chemotherapy could be applied instead.

Neoadjuvant chemotherapy aims to reduce or at least stabilize the cancer until fetal maturity, with final treatment after the delivery. Chemotherapy is allowed from 12 to 14 weeks' gestational age onwards. ${ }^{7}$ In this case, a waiting period of 6 weeks could be used to perform a lymphadenectomy. In case lymph nodes are negative, neoadjuvant chemotherapy could be started with regular clinical and 6-weekly MRI to assess response. This can be continued until fetal viability and ideally until fetal maturity. ${ }^{1}$ Follow-up of tumor growth is important since response is not a guarantee and since progression after initial response can occur.

The decision was made with the patient who decided to undergo a radical hysterectomy with bilateral lymphadenectomy with ovarian preservation and this was performed at 8 weeks' gestation.

\section{Dr Trezza}

At the level of the exocervix, there was an irregular area with ulceration on the surface measuring $2.5 \times 1.5 \mathrm{~cm}$, with $<1 / 3$ invasion of inner myocervix $(25 \%)$. The vaginal cuff $(12 \mathrm{~mm})$, left parametria $(20 \times 15 \mathrm{~mm})$, and right parametria $(20 \times 16 \mathrm{~mm})$ were all negative for disease. In addition, lymph nodes were all negative for disease (four left and four right). The endometrium showed a polypoid formation of $8 \mathrm{~mm}$ of irregular shape, congestion, and edema (Figure 3A-C).

In the Tumor Committee, considering the pathological findings, it was decided that the patient did not require any further treatment and to continue surveillance with a gynecologic oncologist. The patient has been followed for 29 months and is free of disease.

\section{CASE 3}

A 31 -year-old patient $G_{1} P_{0}$ presented at 13 weeks' gestation to a private medical center with abnormal bleeding. On physical examination, there was an exophytic cervical tumor (size not reported from outside medical report) and a biopsy revealed a grade 2 , invasive squamous cell carcinoma. The patient was referred to the University Hospital of Maternity and Neonatology at 24 weeks' gestation. There was no intervention performed in the interim, given lack of expertise in the town and limited means of the patient to travel to our center. Past medical history was significant for gestational diabetes and preeclampsia. Physical examination at the time of presentation in our institution showed an exophytic tumor of approximately $6 \mathrm{~cm}$ in diameter with bilateral parametrial involvement (FIG0 2018 Stage IIB).

\section{Dr Trezza}

Cervical biopsy showed a moderately differentiated invasive squamous cell carcinoma with neoplastic epithelial cell cords and nests (Figure 4A,B).

A pelvic MRI (without contrast) was performed at 22 weeks which showed a cervical lesion in the anterior-lateral region of the vagina, in intimate contact with the bladder. The lesion measured $5.6 \times 4.5 \times 7.0 \mathrm{cms}$ in diameter. There was also evidence of bilateral parametrial involvement. No evidence of pelvic adenopathy was noted, and small and multiple nonspecific bilateral inguinal lymph nodes were identified. No evidence of disease was noted in the rectosigmoid junction (Figure 5A\&B). 


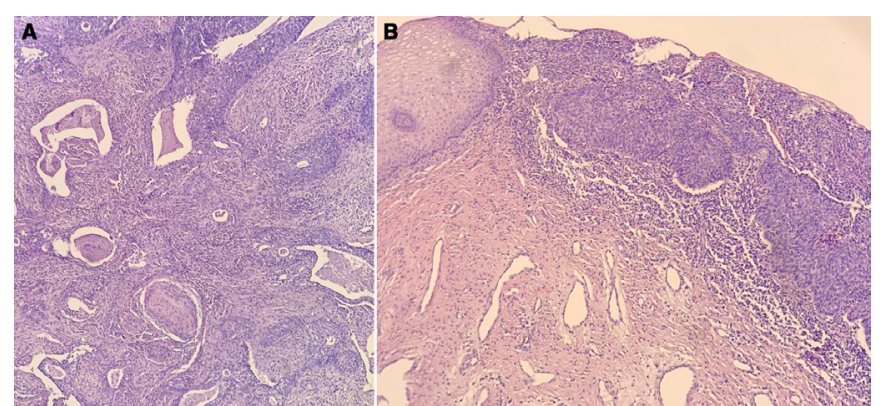

Figure $4(A, B) H \& E$ biopsy of a grade 2 , invasive squamous cell carcinoma.

The patient was discussed at the Tumor Committee. The following options were discussed with the patient: chemotherapy in the form of single-agent cisplatin followed by Cesarean section at 32 weeks if there was tumor response vs expectant management until fetal maturity followed by Cesarean section and subsequent chemotherapy with radiation. The patient decided to maintain her pregnancy without any treatment until delivery.

\section{Dr Amant: At this point based on the diagnosis and gestational age of the patient what would be your recommendations for the patient? Also, what would be your recommended surveillance given that the patient opted for no treatment during the pregnancy?}

Here, we deal with a patient with cervical cancer stage IIB at a gestational age of 24 weeks. Apart from the cervical cancer, she has a high-risk pregnancy due to a history of gestational diabetes and preeclampsia. Also, her socioeconomical situation is poor.

First of all, the pregnancy is too advanced to terminate. Regarding therapeutic options, only a conservative option or chemotherapy remain. Indeed, the cancer is not only too large to perform any surgery at the level of the cervix, also a pelvic lymphadenectomy at this gestational age is rendered technically difficult. ${ }^{14}$ The size of the uterus is too large to guarantee an adequate resection of lymph nodes. Personally, I would recommend combination chemotherapy carboplatin-paclitaxel in a weekly or 3-weekly schedule, until fetal viability or preferentially until fetal maturity. Combination chemotherapy is more likely to obtain a good response in contrast to single-agent chemotherapy. Also, I prefer not to use cisplatin since the transplacental passage is high $(57 \% \text { of maternal levels })^{8}$ and since it can be associated with hearing loss in the offspring. ${ }^{9}$ We know that carboplatin is equally effective in cervical cancer. Long-term follow-up data show that children perform like their age- and gender-matched controls..$^{10-12}$ However, a poor

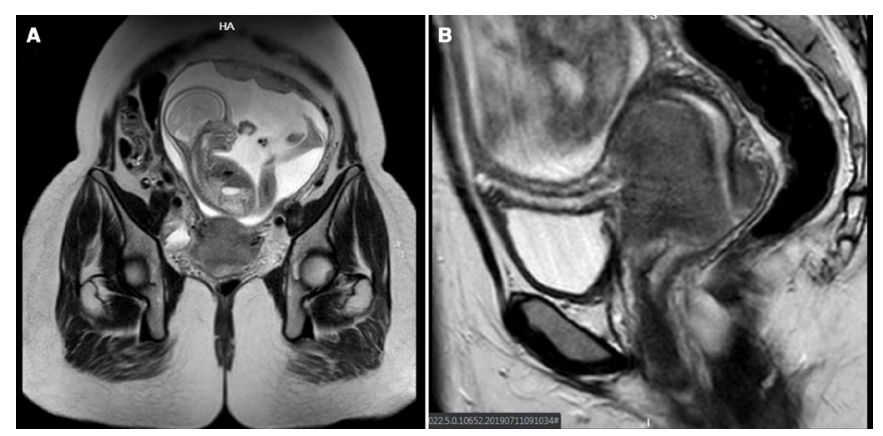

Figure 5 (A,B) Pelvic MRI (without contrast) at 22 weeks' gestation. maternal outcome is linked to a lower verbal IQ, necessitating educational attention in the offspring. ${ }^{12}$ Where possible so when the cancer is responding to the chemotherapy and in this case when there are no concurrent gestational complications, I would continue with chemotherapy beyond 32 weeks for the benefit of the fetus who will then suffer less from prematurity. Follow-up of fetal growth is important since this may be impaired by chemotherapy. Therefore, obstetricians and/or perinatologists should be part of the multidisciplinary team. ${ }^{13}$

In case the patient prefers an expectant approach, surveillance is by speculum examination or MRI. ${ }^{14}$ In case of the absence of progression during an expectant management, the delivery could be scheduled beyond 32 weeks in order to have less prematurity. An unresolved question is how to deal with progression: when is the progression so important that an expectant management is no longer safe? The use of chemotherapy is the best way to avoid this scenario.

A Cesarean section was performed at 32 weeks' gestation. A male child was delivered (Apgar 6/7, weight $1720 \mathrm{gm}$, gestational age by Capurro score 30 weeks). Six weeks after surgery, the patient was referred to another institution where right nephrostomy was performed due to an acute obstructive renal failure. The patient underwent external beam radiotherapy and brachytherapy, given that chemotherapy was not feasible because of the acute kidney failure. After 4 months, the acute obstructive renal failure resolved. The patient is undertaking oncology and nephrology follow-ups. Radiotherapy was completed in January 2020. In April 2020, the pelvic examination showed an atrophic vagina and normal cervix.

\section{Dr Amant: What should be the considerations when} administering chemotherapy during pregnancy, particularly addressing treatment during each trimester of pregnancy? Although it is counterintuitive to administer a drug that is designed to kill rapidly dividing cells during pregnancy, chemotherapy after the first trimester of pregnancy is safe. Indeed, there are no other or more congenital malformations when chemotherapy is administered after 12-14 weeks' gestational age. ${ }^{15}$ However, when given during the first trimester of pregnancy, these drugs increase the chance for congenital malformations and as such are the contraindicated until a gestational age of 12 weeks. ${ }^{7}$ Where possible, an additional 2-week safety period is used since the exact duration of pregnancy is not always certain.

Please provide information on the mode of delivery of the patient and when performing a Cesarean section as to what should be the appropriate incision on the skin and in the uterus.

In the presence of the cervical cancer, a Cesarean section is mandatory since a well perfused episiotomy site at body temperature is an ideal environment for cancer cell growth. Indeed, fatal episiotomy recurrences have been described. ${ }^{1}$ In addition, I prefer to perform a midline skin incision providing a corporeal uterine incision allowing me to stay away from the cervical cancer and avoid abdominal scar metastases.

What should be the ideal mode of surveillance for patients diagnosed with cervical cancer in each of the trimesters? There are no studies that explored the ideal mode of surveillance. Clinicians want to be informed about tumor response during chemotherapy or velocity of progression in case of an expectant management. We mostly perform a speculum examination every 


\section{Case study}

3 weeks. Regarding imaging modalities, a CT scan is not used since it is associated with radiation and less informative when compared with MRI. This latter modality is not associated with ionization and is the standard examination for cervical cancer assessment outside pregnancy. MRI may be used in all trimesters of pregnancy and is informative about the cervical cancer without the use of contrast agents. ${ }^{14}$ In the absence of any data, we perform an MRI every two cycles of 3-weekly chemotherapy or bi-monthly in case of an expectant management. It is important to stress that tumor progression can occur after initial chemotherapy-induced tumor regression. Thus, surveillance is indicated until delivery.

\section{CLOSING SUMMARY}

Cervical cancer is the most frequent malignancy diagnosed during pregnancy and fortunately most patients are diagnosed at an early stage. Management of cervical cancer during pregnancy depends on a number of factors such as the stage of disease (tumor size), lymph node involvement, duration of pregnancy, histological subtype, the patient's desire to continue the pregnancy, and desire for future childbearing. It is important to highlight that during pregnancy the oncologic outcome of cervical cancer patients is like that of nonpregnant patients, noting that the impact of pregnancy on tumor biology remains unclear. A delay in treatment to achieve fetal viability or to improve outcome of the fetus may be an option for patients with early-stage cervical cancer.

Standard treatment for patients with locally advanced cervical cancer remains chemotherapy and radiation. Since radiation therapy may be associated with spontaneous abortion, congenital malformations, and pediatric malignancies it is not routinely recommended during pregnancy. An expectant management may be feasible for patients already near term, as the fetus has already reached lung maturity and the risk of perinatal morbidity is low. However, treatment delays in patients with locally advanced disease may adversely impact maternal morbidity and mortality from disease progression. Neoadjuvant chemotherapy has emerged as an alternative to concurrent chemotherapy and radiation, and it may offer patients the option to delay definitive treatment until fetal viability. In this setting, platinumbased chemotherapy is the most frequently used systemic therapy in pregnant women. One should note that chemotherapy administered during the second and third trimester may be associated with intrauterine growth retardation, prematurity, and low birthweight. In addition, the administration of chemotherapy within 3 weeks of anticipated delivery is not recommended.

In terms of mode of delivery, the literature is mixed as it pertains to the ideal approach and thus several items need to be considered. Most patients undergo Cesarean section. However, it seems that planned vaginal delivery in the setting of microinvasive disease or vaginal delivery in the absence of a confirmed diagnosis is not associated with disease progression, obstetric complications, or decreased survival.

Cervical cancer diagnosed in the context of pregnancy remains a rare event. It is imperative that all pregnant patients undergo screening evaluation, and that maternal fetal medicine and gynecologic oncology teams work together to ensure for the implementation of strategies to maximize a favorable outcome for both the mother and the baby. Counseling of patients by a multidisciplinary team is paramount.

Correction notice This article has been corrected since it first published. The provenance and peer review statement has been included.

Funding The authors have not declared a specific grant for this research from any funding agency in the public, commercial, or not-for-profit sectors.

Competing interests None declared.

Patient consent for publication Not required.

Provenance and peer review Commissioned; internally peer reviewed.

\section{REFERENCES}

1 Amant F, Berveiller P, Boere IA, et al. Gynecologic cancers in pregnancy: guidelines based on a third International consensus meeting. Ann Oncol 2019;30:1601-12.

2 Morice P, Uzan C, Gouy S, et al. Gynaecological cancers in pregnancy. Lancet 2012;379:558-69.

3 Maggen C, Wolters V, Cardonick E, et al. International Network on Cancer, Infertility and Pregnancy (INCIP): pregnancy and cancer: the INCIP project. Curr Oncol Rep 2020;22.

4 Halaska MJ, Uzan C, Han SN, et al. Characteristics of patients with cervical cancer during pregnancy: a multicenter matched cohort study. An initiative from the International network on cancer, infertility and pregnancy. Int J Gynecol Cancer 2019;29:676-82.

5 Van Calsteren K, Hanssens M, Moerman P, et al. Successful conservative treatment of endocervical adenocarcinoma stage IB1 diagnosed early in pregnancy. Acta Obstet Gynecol Scand 2008;87:250-3.

6 Ramirez PT, Pareja R, Rendón GJ, et al. Management of low-risk early-stage cervical cancer: should conization, simple trachelectomy, or simple hysterectomy replace radical surgery as the new standard of care? Gynecol Oncol 2014;132:254-9.

7 Cardonick $E$, lacobucci $A$. Use of chemotherapy during human pregnancy. Lancet Oncol 2004;5:283-91.

8 Calsteren KV, Verbesselt R, Devlieger R, et al. Transplacental transfer of paclitaxel, docetaxel, carboplatin, and trastuzumab in a baboon model. Int J Gynecol Cancer 2010;20:1456-64.

9 Geijteman ECT, Wensveen CWM, Duvekot JJ, et al. A child with severe hearing loss associated with maternal cisplatin treatment during pregnancy. Obstet Gynecol 2014;124:454-6.

10 Amant F, Vandenbroucke $T$, Verheecke $M$, et al. International Network on Cancer, Infertility, and Pregnancy (INCIP): pediatric outcome after maternal cancer diagnosed during pregnancy. $N$ Engl J Med 2015;5;373:1824-34.

11 Vandenbroucke T, Verheecke M, Fumagalli M, et al. Effects of cancer treatment during pregnancy on fetal and child development. Lancet Child Adolesc Health 2017;1:302-10.

12 Vandenbroucke T, Verheecke M, van Gerwen M, et al. International Network on Cancer, Infertility and Pregnancy (INCIP): child development at 6 years after maternal cancer diagnosis and treatment during pregnancy. Eur J Cancer 2020;138:57-67.

13 de Haan J, Verheecke M, Van Calsteren K, et al. International Network on Cancer and Infertility Pregnancy (INCIP). oncological management and obstetric and neonatal outcomes for women diagnosed with cancer during pregnancy: a 20-year international cohort study of 1170 patients. Lancet Oncol 2018;19:337-46.

14 Vandecaveye V. Imaging modalities in pregnant cancer patients in press IJGC, 2020.

15 Van Calsteren K, Heyns L, De Smet F, et al. Cancer during pregnancy: an analysis of 215 patients emphasizing the obstetrical and the neonatal outcomes. JCO 2010;28:683-9. 